\title{
Sleep and the ICU
}

\author{
Janice Wang ${ }^{*}$ and Harly Greenberg
}

Division of Pulmonary, Critical Care and Sleep Medicine, Hofstra North Shore-LIJ School of Medicine, New Hyde Park, USA

\begin{abstract}
Disturbed sleep is common in critical illness, not only during early phases of treatment in an intensive care unit (ICU) but also during later stages of recovery after ICU discharge. While sleep quality during critical illness is usually not a primary concern of intensivists, disrupted sleep can impede recovery and has been associated with immune system dysfunction, impaired wound healing, and adverse neurological and psychological outcomes [1]. The noise and lighting of the ICU environment, frequent patient-provider interactions, and critical care procedures all profoundly impact sleep quality and continuity in critically ill patients. Various sedative medications and various modes of mechanical ventilation can also affect sleep. This article will review (1) sleep disruption and its clinical manifestations in the ICU patient, (2) the effects of the ICU environment and routine critical care on sleep disturbances, (3) the biological consequences of critical illness on sleep and circadian rhythms, and how sleep deprivation (SD) affects the immune system, (4) iatrogenic disturbances of sleep in the ICU patient (e.g. commonly used sedatives, mechanical ventilation), and (5) sleep during recovery after critical illness.
\end{abstract}

Keywords: ICU and sleep disturbances, ICU and circadian rhythms, sleep deprivation and immune system, ICU and mechanical ventilation.

\section{SLEEP DISRUPTION AND ITS CLINICAL MANIFESTATIONS IN THE ICU PATIENT}

Several studies have used polysomnography to objectively determine the characteristics and quantity of sleep in the ICU patient. Surprisingly, total sleep time achieved over the course of a 24 hour period in critically ill patients approaches normal values [2]. However, sleep continuity and sleep architecture are markedly perturbed. Nocturnal sleep is often severely reduced with approximately 50 percent of sleep occurring during daytime hours. When sleep ensues, it usually occurs in short, highly fragmented bouts [2-4]. Clinical severity of illness assessment, using the simplified acute physiology score-II $\left(\mathrm{SAPS}_{\mathrm{II}}\right)$, has been shown to positively correlate with the amount of sleep that occurs during daytime hours based on objective measures [5]. However, another study using acute physiology and chronic health evaluation scores (APACHE II and APACHE III) found no association between severity of illness and subjective self-reports of sleep quality. It is worth noting, however, that subjective reports of sleep quality in the ICU may be erroneous because of misperception of sleep, possibly related to sedative medications and effects of critical illness on cognitive function that might alter recall of sleep quantity and quality. Objective studies of sleep quality in a critical care setting using polysomnographic analysis revealed that nocturnal sleep is highly fragmented with frequent arousals and

*Address correspondence to this author at the Division of Pulmonary, Critical Care and Sleep Medicine, Hofstra North Shore-LIJ School of Medicine, 410 Lakeville Road, Suite 107, New Hyde Park, NY 11040, USA; Tel: 516-465-5400; Fax: 516-465-5454;

E-mail: janiceywang@gmail.com awakenings. In addition, the amount of REM and SWS is severely reduced with an increase in light or stage N1 sleep. Abnormal and disturbed sleep has been documented in virtually all types of critical care settings including medical, surgical and cardiac ICUs. The consequences of sleep abnormalities in critical illness are often overlooked. However, recent data has indicated that fragmented and disturbed sleep in the ICU may adversely affect patient outcome. Increased delirium, irritability, anxiety and depression, decreased pain threshold, increased protein catabolism, hyperglycemia, and immune dysfunction have all been linked to disturbed and insufficient sleep in critical illness [5-7].

\section{THE ICU ENVIRONMENT: IMPACT ON SLEEP AND CIRCADIAN RHYTHMS}

Environmental factors in critical care units that might negatively affect sleep include high noise and light levels at times when sleep is desired. Peak noise in critical care units has been reported to range from an average of 53 to $65 \mathrm{~dB}$ to over $80 \mathrm{~dB}$ throughout the day and night [2-4]. The Environmental Protection Agency recommends that peak noise levels in the ICU should be no higher than 45 decibels (dB) during the day and $35 \mathrm{~dB}$ at night $[2,4]$. Surprisingly, however, noise was documented to be the major cause of sleep disruption in a recent study; loud noises were found to be responsible for only $11.5 \%$ of arousals and $17 \%$ of awakenings, with a mean arousal index of $1.9 \pm 2.1$ arousals/h sleep, in critically ill patients [4]. Gabor et al. also examined the impact of noise in the ICU environment on the sleep of healthy volunteers as well as mechanically ventilated critically ill patients. These investigators also found that noise contributed to approximately $20 \%$ of the observed arousals and awakenings. Healthy subjects actually 
slept relatively well in an open ICU environment with improvement in sleep quantity and quality observed in a single-patient ICU room that allowed for noise reduction.

Aside from noise, other aspects of the ICU environment can disturb the regulation and timing of sleep. Current concepts regarding the control of sleep and wakefulness encompass two major factors, homeostatic drive and circadian rhythms. Homeostatic drive, which is determined by the amount of prior wakefulness, influences the amount and depth of sleep. Circadian rhythms regulate the timing of sleep and wakefulness, in addition to many other biological functions [8]. In most humans, the tau, or daily cycle of circadian rhythms, is slightly longer than 24 hours (Fig. 1). As a result, circadian rhythms must be realigned with the Earth's day-night cycle on a daily basis. Failure to achieve this realignment can result in a "free running" rhythm where sleep and wakefulness become progressively delayed each day. Misalignment of the timing of sleep and wakefulness ensues which can ultimately progress to complete reversal of the day night cycle. Various environmental cues, including bright light exposure, feeding and other behavioral functions can serve as "zeitgebers" or stimuli, which reset or realign circadian rhythms to coincide with the day-night schedule. However, patients in critical care settings are often deprived of appropriately timed zeitgeibers such as bright light and food administration. Exposure to sunlight is limited or nonexistent in critical care units and most artificial lighting is of insufficient intensity to serve as a zeitgeber that fully realigns circadian rhythms. However, inappropriate timing of light exposure such as during the night, even at lower intensities, can impact the circadian pacemaker and produce an adverse effect on sleep timing. Nocturnal light levels are variable in ICUs, ranging from less than 5 lux to more than 1400 lux [2]. Light levels of 300 to 500 lux can affect the human circadian pacemaker and have the potential to impair nocturnal sleep. Appropriately timed meals, which are also potent zeitgebers, are often replaced by continuous feeding in the ICU (e.g. tube feeds or total parenteral nutrition), thus eliminating yet another potential means of maintaining appropriate alignment of circadian rhythms. As a result, misalignment of sleep and wakefulness with the day-night cycle is common in critically ill ICU patients.

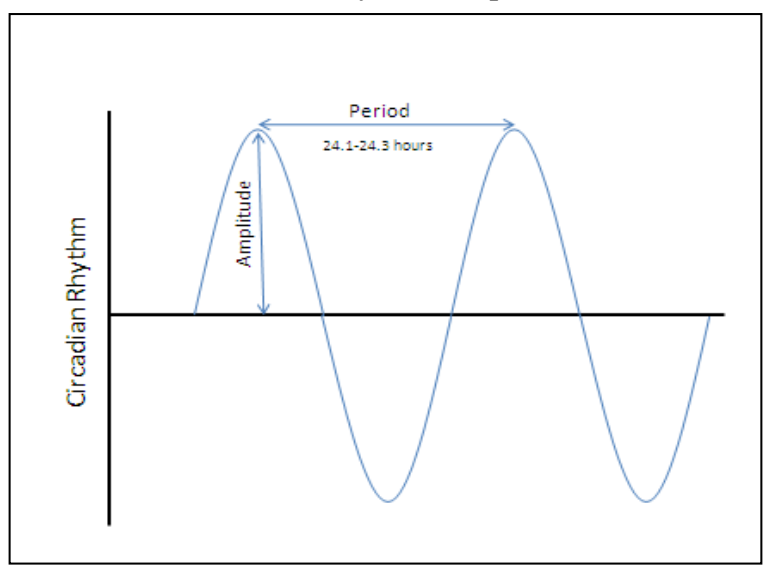

Fig. (1). The human circadian clock has a free-running period, or tau, of 24.1-24.3 hours.

Patient care activities, such as nocturnal nursing assessments, vital sign checks, neurologic evaluations, IV pump monitoring, medication administration and bathing all have the potential to disturb sleep. Le, et al. assessed the effect of patient-care activities on sleep continuity in the ICU. Surprisingly, only $7 \%$ of sleep disturbances could be directly linked to these activities in medical, surgical, cardiothoracic, pediatric, and neonatal ICUs [9]. For unclear reasons, the surgical ICU had the most frequent nocturnal nursing interactions, the most common of which was nursing assessments. Interestingly, the nursing staff estimated that $13.9 \%$ of all nocturnal interactions could be safely omitted without adversely affecting patient care, thus lessening the number of sleep disruptions at night. While ICU noise, high light levels and patient-care activities at night individually account for only a small percentage of sleep disruptions, collectively these factors can lead to significant disturbances of sleep and may contribute to sleep deprivation. Reduction of these disturbances has the potential to improve sleep quality in critical care units.

Delirium is a common complication in the ICU characterized by a fluctuating course of shifting attention, disorganized thinking, confusion, and an altered level of consciousness [10]. Acute illness and sensory overload in the setting of the ICU environment can be very stressful and can result in delirium even in an individual with normal baseline cognition. Sleep fragmentation and deprivation may increase the risk of developing delirium [11]. Severe REM deprivation, defined as REM sleep that encompasses less than $6 \%$ of total sleep time (normal $15-25 \%$ ) has also been associated with a higher incidence of delirium compared with patients who exhibit more REM sleep [11]. Delirium is a strong predictor of mortality, time on mechanical ventilation, length of ICU stay and post-ICU hospitalization. Delirium occurring during critical illness also increases risk for subsequent functional decline [12-14]. A randomized controlled trial that investigated the use of nighttime earplugs in ICU patients to reduce noise-induced sleep fragmentation studied 69 patients sleeping with earplugs during the night compared to a control group sleeping without earplugs. The Neelon and Champagne Confusion Scale (NEECHAM), a validated scoring system for confusion and delirium, was used to assess each patient. The intervention group scored $15 \%$ in the mild confusion category whereas the control group scored $40 \%$. Combining assessments of both mild confusion and delirium demonstrated cognitive disturbances in $35 \%$ of the study group, with a hazard ratio of 0.47 (95\% confidence interval of 0.27 to 0.82 ) versus $60 \%$ in the control group. When delirium or confusion occurred, onset was delayed compared to patients who did not use earplugs. ${ }^{10}$ Patients who used earplugs also reported better sleep quality; nearly half of the study group reported a better sleep perception compared to $25 \%$ of the control group. While another randomized controlled trial which also investigated the effect of nocturnal earplug use in the ICU did not demonstrate a decrease in the incidence of delirium, in that study earplug use was associated with less confusion and cognitive impairment as well as improved self-reported sleep quality [15]. Thus, a relatively simple measure that reduces the impact of ICU noise can improve sleep quality and may improve cognition in critically ill patients. 


\section{CIRCADIAN RHYTHMICITY IN THE ICU PATIENT}

As mentioned above, circadian rhythms orchestrate the timing of sleep and wakefulness in addition to regulating many other biological processes. Normal amplitude, periodicity and timing of circadian rhythms can be essential to normal physiology, both at the organ system and cellular levels. Past studies have demonstrated loss of intrinsic circadian rhythmicity in critically ill patients [16, 17]. However, confounding factors that may have affected the validity of this conclusion include varying severities of illness and use of vasopressor agents with sympathomimetic activity which could elevate melatonin levels which, in turn, might alter intrinsic circadian rhythms [18]. More recent evidence obtained from studies of $24 \mathrm{hr}$ urinary excretion of the melatonin metabolite, 6-sulfatoxymelatonin (aMT6), in mechanically ventilated patients sedated with propofol and/or a benzodiazepine demonstrated preserved circadian rhythmicity in this critically ill cohort, Fig. (2) [8]. However, the timing of the circadian rhythm of melatonin excretion was abnormal with a phase delay of the rhythm.

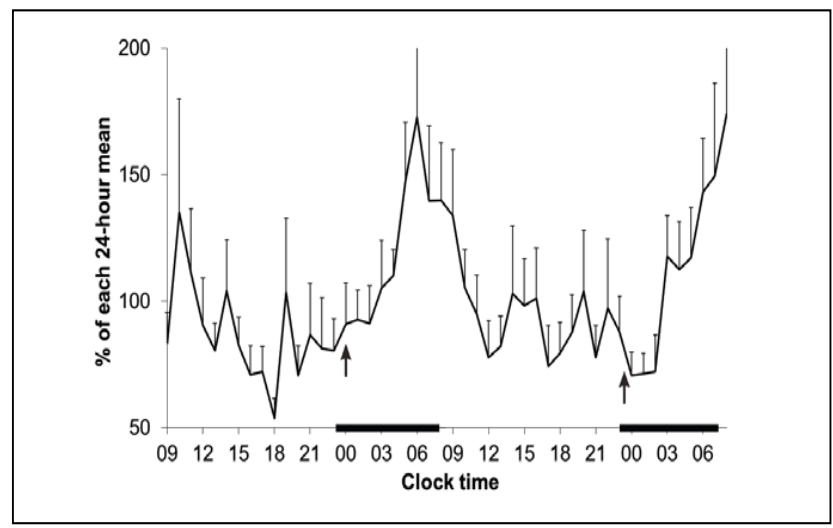

Fig. (2). Temporal profile of 6-sulfatoxymelatonin (aMT6) excretion in mechanically ventilated patients over 2 days demonstrating overall preservation of the circadian rhythm of aMT6s excretion. The black bars denote usual sleep time (23: $00-$ 07: 00). Reproduced with permission from Sleep [8].
The observation of a phase delay of the melatonin rhythm is suggestive of a "free running" state where circadian rhythms are not entrained to the timing of the day-night cycle by usual "zeitgebers" or environmental cues. Because the periodicity of human circadian rhythms is typically slightly longer than $24 \mathrm{hrs}$, a gradual and progressive delay of the timing of circadian rhythms occurs each day in the absence of entraining "zeitgebers", which can result in the observed phase delay, (Fig. 3) [19]. Disturbances of circadian rhythms may be most pronounced in patients with traumatic brain injury which might be associated with damage to the suprachiasmatic nucleus, the master regulator of circadian rhythms, or its CNS projections [20].

As mentioned, the timing and progression of sleep across the night is regulated, in part, by circadian rhythms. Normally, sleep progresses in three to five 90 minute nonrapid eye movement (NREM) / REM sleep cycles across the night, with slow wave activity predominating in the first third of the night and REM sleep encompassing a large part of the last third of sleep. This normal sleep "architecture" as depicted in Fig. (4) was absent in a study of critically ill patients.

In fact, temporal dissociation of EEG slow wave activity (SWA) from the biological night, as defined by the circadian rhythm of melatonin excretion, was evident. Rather than occurring as it normally does in the first third of the night, SWA that was observed in these critically ill patients occurred at any time during the day and night. In addition, REM sleep was virtually absent in this cohort. The clinical implications of this "uncoupling" of the circadian timing system from the sleep cycle are unclear [8]. However, secretion of norepinephrine, prolactin, cortisol, growth hormone and other hormones and biological processes that are affected by sleep and circadian rhythms, may be adversely affected in critically ill patients. Normal variation in levels or pulsatile secretion may be lost [2].

The function of the hypothalamic-pituitary-adrenal axis (HPA), which plays a major role in the response to stress, is affected by critical illness. Activity of the HPA axis can be assessed by plasma levels of adrenocorticotropic hormone

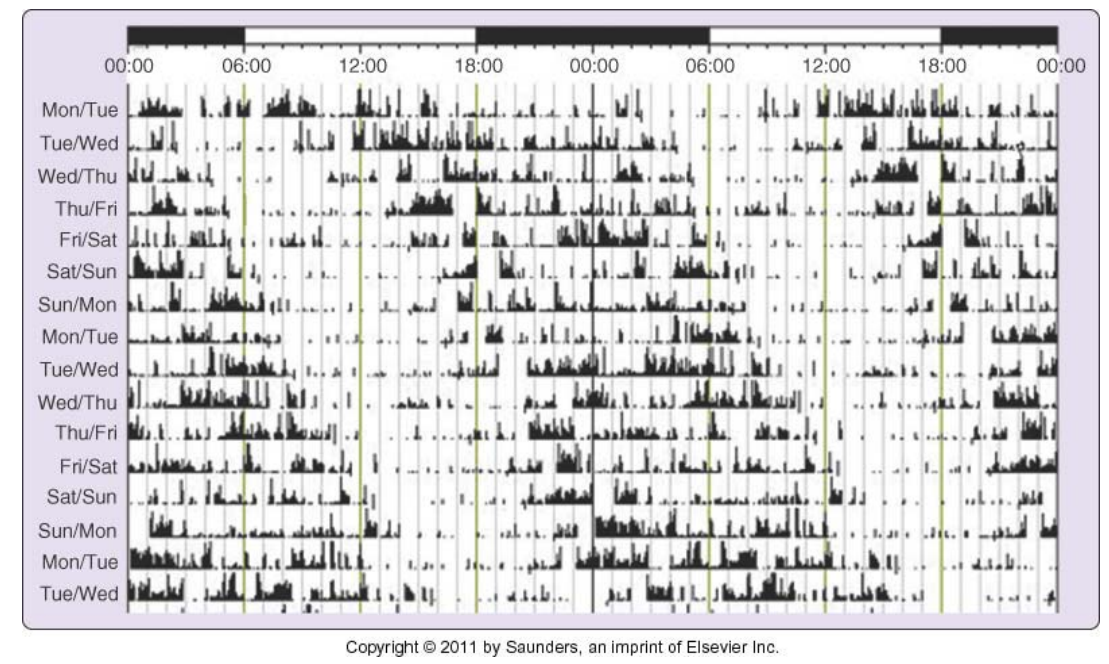

Fig. (3). A characteristic actogram seen in free-running circadian disorder and also in the absence of entraining zeitgebers. Sleep onset becomes later on each consecutive day. The black bars indicate activity levels recorded by wrist monitor [19]. Reproduced with permission from Elsevier Science. 


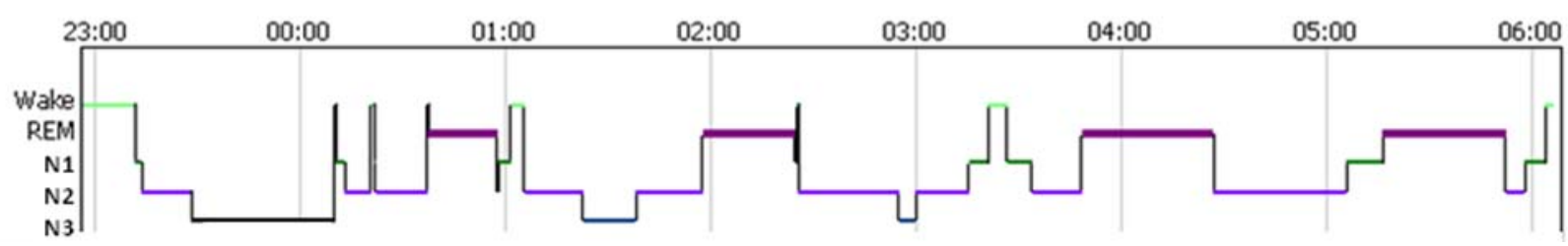

Fig. (4). Histogram depicting the progression of sleep stages across a single night in a normal adult.

(ACTH) and cortisol. Normally, ACTH and cortisol levels are highest during early morning hours and decline throughout the day, reaching a nadir in late evening (Fig. 5).

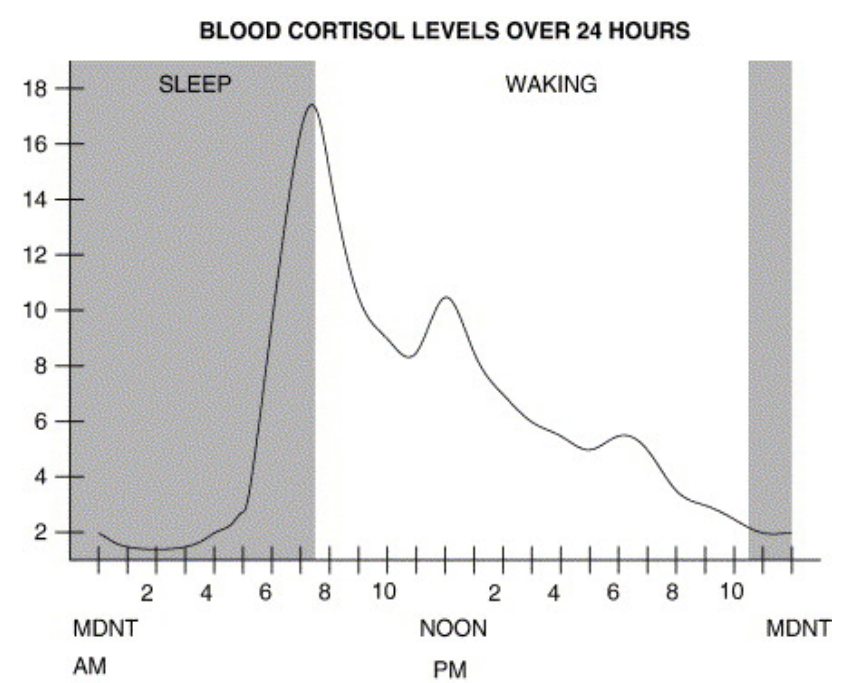

Fig. (5). The 24-h plasma cortisol secretion curve in humans peaks near the time of awakening and has a nadir during the early part of the sleep cycle. Lovallo W. Cortisol secretion patterns in addiction and addiction risk. Int J Psychophysiol. 2006; 59: 195-202. Reproduced with permission from Elsevier Science.

During sleep deprivation, the nadir of cortisol secretion is higher due to the absence of inhibitory effects of the first hours of sleep; morning cortisol levels are lower, due to absence of the stimulating effects of morning awakening [19]. Several factors can influence the activity of the HPA axis in critical illness. Disturbances of circadian rhythms, sleep deprivation, stress, shock as well as adrenal insufficiency may all contribute to abnormal HPA function. For example, absence of the normal variation in cortisol levels over a 24-hour period is commonly observed in critical illness. The ICU environment itself, filled with noise and nocturnal lighting, increases urinary excretion of cortisol at night; restoration of normal circadian rhythmicity of cortisol secretion has been demonstrated to be reversible with earplugs and eyemasks in one ICU study [21].

\section{SLEEP, INFLAMMATION AND IMMUNITY}

Sepsis is one of the most common reasons for ICU admission and has tripled in incidence over the past two decades in the United States [22]. ICU patients are also at risk for hospital-acquired infections. Sepsis is believed to adversely affect sleep; it is associated with increased NREM and decreased REM sleep [2]. Increased levels of the cytokines interleukin (IL)-1, IL-6 and tumor necrosis factor (TNF) $\alpha$ are observed in infection and sepsis. These cytokines have sleep promoting properties and can induce excessive daytime sleepiness, fatigue and reduced cognition $[23,24]$. The sleep promoting properties of IL- 1 and TNF $\alpha$ were demonstrated in a study that showed increased amplitude of delta or SWA after central injections of IL-1 or $\mathrm{TNF} \alpha$ in rabbits in a dose-dependent manner [24]. The observed increase in SWA was similar to that seen after recovery from sleep deprivation. Higher doses of these cytokines further enhance NREM sleep at the expense of REM sleep. At the highest doses, both NREM sleep and REM sleep are inhibited in association with occurrence of high fever [24].

As mentioned, circadian rhythmicity may be diminished or entirely lost in critically ill patients. Melatonin in a healthy individual is normally inhibited by light during the daylight hours, and its secretion increases approximately 2 to 3 hours before habitual bedtime in the presence of dim light, called the time of dim light melatonin onset (DLMO). Melatonin levels then peak between 1 am and $3 \mathrm{am}$. The pattern of melatonin secretion has been used as a marker of circadian rhythmicity [25]. A normal pattern of melatonin secretion is typically maintained in nonseptic ICU patients, however in sepsis, DLMO and peak of melatonin secretion is lost, resulting in a continuous stimulation of melatonin release [17]. Additionally, in septic patients, melatonin secretion remained abnormal for several weeks after recovery from sepsis [17]. Recognition of the implications of circadian disturbance is essential as further evidence suggests the existence of circadian-immune interactions [26]. The mouse spleen, thymus, inguinal lymph nodes and peripheral blood exhibit clock gene expression [27]. Human $\mathrm{CD} 4^{+} \mathrm{T}$-cells possess a functional molecular clock and show circadian variations of IL-2, IL-4, and IFN- $\gamma$ production [28]. The clinical implications of abnormalities of circadian rhythms on immune function have not been fully elucidated and are difficult to entirely separate from effects of sleep deprivation.

Immune function is vital for recovery and while sepsis itself may disturb sleep, sleep deprivation has been shown to adversely affect the immune system [29-31]. Evidence suggests that sleep may be vital to basic host defense mechanisms. Ruiz et al. studied the effects of total sleep deprivation (SD) and REM SD on white blood cell (WBC) count, T- (CD4/CD8) and B-lymphocytes, Immunoglobulin (Ig) classes, and complement and cytokine measurements in healthy subjects. Subjects were randomly assigned to protocols of control, two nights of total SD, or four nights of REM SD. Sleep deprived subjects then underwent three nights of sleep recovery. SD may be associated with a proinflammatory state: WBC counts increased by $15 \%$ from baseline after total SD, with neutrophils increasing by $15 \%$ as well; counts returned to baseline after one night of recovery. Also, supporting the hypothesis that an inflammatory state is associated with SD, Meier-Ewert et al. demonstrated that the 
inflammatory marker C-reactive protein increased during total and partial SD in healthy subjects [32]. The effects of $\mathrm{SD}$ on $\mathrm{CD}^{+}$T-cells and IgA levels suggest that adapative immunity may be more vulnerable to SD [30]. CD4 ${ }^{+}$T-cells increased during total SD and did not return to baseline after three nights of sleep recovery. IgA levels decreased during REM SD. Counts of CD8 ${ }^{+} \mathrm{T}$-cells, total lymphocytes, and Bcells were not significantly different from baseline after SD. Complement and cytokine levels (IL-1 $\beta$, IL-2, IL-4, IL-6, IL-10, TNF- $\alpha$, and interferon- $\gamma$ ) remained unchanged after SD.

Cellular immunity against viral infections may also be impaired by SD; natural killer cell activity was reduced to $72 \%$ below mean baseline lytic activity in healthy subjects after partial SD [31]. In response to influenza vaccination, healthy subjects whose sleep was restricted to 4 hours for 6 nights after vaccination had less than half the level of antibody titers compared to subjects who slept 7.5 to 8.5 hours per night for ten days after vaccination, although by 3 to 4 weeks after vaccination these differences resolved [33]. Thus, sleep may play an important role in the formation of immunological memory. It has been proposed that the adverse effects of SD on the immune system may be severe enough to impair wound healing [34, 35].

Many studies on the effects of sleep deprivation on immune function evaluated the effects of relatively brief periods of acute sleep deprivation. However, prolonged periods of sleep loss or sleep restriction, during a prolonged ICU stay for example, represent a condition of prolonged stress. Similar to acute studies, prolonged periods of sleep deprivation also lead to a pro-inflammatory state with increased plasma concentrations of IL-6 and the soluble TNF- $\alpha$ receptor 1 . Ten days of sleep restriction to $4 \mathrm{~h}$ per night increases the concentration of $\mathrm{C}$-reactive protein. Thus, chronic sleep deprivation also appears to induce a state of systemic inflammation along with impairment of immune responsiveness [36].

\section{EFFECTS OF MEDICATIONS ON SLEEP IN THE ICU}

Medications, especially sedatives, can have a significant impact on sleep and can affect the course of an ICU patient's hospitalization. These medications can suppress slow wave and REM sleep and a rebound increase in these sleep stages may occur after drug withdrawal. Awareness of these effects is useful when assessing mental status. Patients sedated with propofol or midazolam infusions may subjectively report better sleep quality than non-sedated patients $[6,37]$. The sedated patients' perception of better sleep quality may be a consequence of reduced discomfort associated with intubation and mechanical ventilation and also limited recall while under sedation [6]. However, it is important to recognize the similarities as well as important differences between sleep and sedation. Similarities between sleep and sedation include motor hypotonia, temperature dysregulation, disconjugate eye movements, altered sensorium and mentation, and respiratory depression [2]. Sleep, however, is regulated, in part, by circadian rhythms and demonstrates an ultradian pattern with progression of sleep stages in 90 minute cycles of NREM and REM sleep as evidenced by specific patterns of brain activity recognized on EEG recordings. Sleep is also associated with decreased norepinephrine release from the wake-promoting locus coeruleus neurons. In contrast, sedation can alter circadian rhythmicity and typically is not associated with suppression of norepinephrine release from the locus coeruleus [2].

Propofol, a $\mathrm{GABA}_{\mathrm{A}}$ agonist, is a commonly used sedative in the ICU. In a randomized crossover study of mechanically ventilated ICU patients with or without propofol, those sedated with propofol demonstrated worsening sleep quality, characterized by a lack of sequential progression through sleep stages and reduced SWS and REM sleep [38]. In this study, respiratory variables, patient-ventilatory asynchrony, and arterial blood gases did not differ with or without propofol, suggesting that propofol has a significant effect on sleep even when controlling for confounding variables. Dexmedetomidine, a selective alpha 2-adrenergic receptor agonist, is used for its light sedative effects in the ICU. It acts primarily in the locus ceruleus by inhibiting wakepromoting norepinephrine pathways, thereby depressing alertness and sympathetic activity [13]. Dexmedetomidine also suppresses wake-promoting activity of the histaminergic tuberomammillary nucleus [13]. EEG activity during dexmedetomidine sedation appears to resemble NREM sleep with abundant sleep spindle and slow-wave activity. These findings support the hypothesis that this sedative preserves or promotes non-rapid eye movement sleep [39].

Other commonly used medications in the ICU include benzodiazepines, opioids, and vasopressors. Benzodiazepines, acting through $\mathrm{GABA}_{\mathrm{A}}$ receptors, and opioids, through $\mu$ receptors, are known to suppress REM and SWS [40]. Benzodiazepines have high affinity for multiple $\mathrm{GABA}_{\mathrm{A}}$ receptor subunits. Activation of the $\alpha 1$ subunit induces sedative effects; binding to the $\alpha 2$ subunit promotes hypnotic properties [14]. Vasopressors such as dopamine, epinephrine, and norepinephrine decrease REM and SWS via adrenergic receptors. Corticosteroids, another commonly used medication in the ICU, decrease REM and SWS [1]. While suppression of specific sleep stages may occur during administration of these medications, a rebound increase in REM or SWS can occur upon drug withdrawal. A rebound increase in REM sleep can be associated with exacerbation of sleep disordered breathing, along with autonomic instability, potentially leading to adverse hemodynamic effects, myocardial ischemia, and altered mentation [41].

A hypnotic class of medication available only in the oral form is the nonbenzodiazepine receptor agonist (e.g. zolpidem and zaleplon). While studies evaluating the role of this class of hypnotic use in the ICU are lacking, they are commonly prescribed to the non-intubated patient and are probably safer than benzodiazepines in the ICU [40]. The nonbenzodiazepine receptor agonists do not possess antiseizure, anti-anxiety, or muscle relaxant properties like the benzodiazepines do. The advantages of this class of hypnotics include less likelihood of causing significant rebound insomnia and tolerance.

\section{EFFECTS OF MECHANICAL VENTILATION ON SLEEP}

Mechanical ventilation typically disturbs and disrupts sleep. Parthasarathy and Tobin studied pressure support ventilation (PSV) and assist-control ventilation (ACV) in 11 critically ill patients, with both modes set to achieve a tidal 
volume of $8 \mathrm{ml} / \mathrm{kg}$ [42]. Sleep fragmentation (i.e. arousals and awakenings) was significantly greater during PSV (79 \pm 7 events $/ h)$ than ACV $(54 \pm 7$ events $/ h)(p=0.02)$. Six of the 11 patients who developed central apneas during PSV were more likely to have heart failure (defined as a left-ventricular ejection of less than $50 \%$ or a history of congestive heart failure) compared to those who did not develop central apneas. Mechanisms responsible for the appearance of central sleep apnea during PSV may involve ventilatory "overshoots", augmented by the pressure support, which drive $\mathrm{PaCO} 2$ below the apneic threshold thus inducing central apneas. This mechanism may be particularly relevant in patients with heart failure and Cheyne-Stokes respiration (CSR) but it may also occur in those without heart failure $[42,43]$. Heart failure patients with CSR have a lower $\mathrm{PaCO} 2$ during wakefulness and NREM sleep than patients without CSR; thus $\mathrm{PaCO} 2$ is closer to the apneic threshold during sleep [44, 45]. The ventilatory instability of CSR results primarily from a high central and peripheral chemoresponsiveness that increases the tendency to hyperventilate, resulting in hypocapnia and a ventilatory overshoot $[44,45]$. Any ventilatory overshoot that occurs after an apnea would be augmented by PSV and may result in hypocapnia that induces further central apneas [42]. When the investigators introduced $100 \mathrm{~mL}$ of dead space to the ventilator circuit of the patients with central apneas, which would be expected to increase $\mathrm{PaCO} 2$, the frequency of central apneas and resultant sleep disruptions was reduced. Of note, ventilator settings are not typically reduced during the night when minute ventilation is typically lower during sleep as compared to wakefulness [42]. This poses an increased risk for inducing hypocapnia, with resultant central sleep apneas, arousals and awakenings. Hypocapnia has also been shown to decrease the amount of REM sleep in animal models in the absence of sleep disordered breathing in both normoxic and hypoxic conditions [46]. Respiratory alkalosis with hypocapnia is associated with less SWS, more arousals, and reduced sleep efficiency. Acute changes in $\mathrm{CO} 2$ and $\mathrm{pH}$ may activate hypothalamic orexin neurons, which are wake promoting, thus further disturbing sleep [5].

Patient-ventilator asynchrony is another factor that may induce sleep disruption. A randomized crossover clinical trial by Bosma, et al. studied 13 patients during weaning from mechanical ventilation on PSV or proportional assist ventilation (PAV) on one night and subsequently crossed over to the alternative mode for the second night [47]. PAV provides pressure in proportion to the inspiratory effort thereby acting as a mode of partial ventilatory support. Without a preset target pressure flow or volume, PAV matches the patient's respiratory efforts, which is especially important when the patient is sleeping; PAV allows for the physiologic increase in $\mathrm{PaCO}_{2}$ during sleep and prevents the development of central apneas and resultant sleep fragmentation [47]. As opposed to PAV, PSV settings do not adapt to the patient's breathing rhythm during periods of wakefulness and sleep. The degree of ventilatory support provided during wakefulness may not be appropriate during sleep. The trial demonstrated that the frequency of patientventilator asynchronies were lower with PAV than with PSV $(24 \pm 15,53 \pm 59$ events/h, respectively; $\mathrm{p}=0.02)$ and correlated with the number of arousals/h. Overall sleep quality was significantly improved on PAV compared to
PSV; there were fewer arousals $/ \mathrm{h}$, fewer awakenings $/ \mathrm{h}$, and greater REM sleep and SWS on PAV than PSV.

While some data suggests that the mode of mechanical ventilator support contributes to sleep fragmentation, as suggested by Parathasarathy and Tobin, it may actually be the settings of the ventilator that are causing the sleep disturbances. Cabello et al. demonstrated that ventilatory mode in nonsedated, conscious ICU patients did not influence sleep pattern, arousals and awakenings, or ineffective efforts when comparisons were made between ACV, clinically adjusted pressure support ventilation (cPSV), and automatically adjusted pressure support ventilation (aPSV) [48]. ACV was adjusted for a tidal volume of $8 \mathrm{~mL} / \mathrm{kg}$ of predicted body weight; cPSV was adjusted by the physician for a tidal volume between 6 and 8 $\mathrm{mL} / \mathrm{kg}$ of predicted body weight and a respiratory rate of $<$ 35 breaths/min; the aPSV adapted the level of PS to keep the patient at a respiratory rate between 15 and 30 breaths $/ \mathrm{min}$, a tidal volume $>300 \mathrm{~mL}$, and end-tidal $\mathrm{CO}_{2}$ level $<55 \mathrm{~mm}$ $\mathrm{Hg}$. Independent of the ventilator mode, sleep architecture was of poor quality, with decreased REM sleep and increased sleep fragmentation. However, on lower levels of PS, these investigators demonstrated that central apneas and ineffective efforts were each responsible for less than $10 \%$ of total sleep fragmentation. These findings suggest that regardless of the ventilatory mode, excessive ventilatory support, resulting in overventilation that contributes to central apneas, is an important factor leading to sleep disruption. Physicians should be aware of iatrogenic causes of central sleep apnea and sleep disturbances in mechanically ventilated patients.

\section{SLEEP AFTER THE ICU}

A majority of patients report improved sleep after leaving from the ICU as a result of reduced nocturnal disruptions and noise, as well as decreased pain and fewer intravenous catheters and tubes [6]. Levels of noise and lighting have been shown to be lower in a step-down unit (SDU) in one study when compared to those of the ICU [5]. However, sleep disturbances may persist after ICU discharge, since many patients are transferred to a SDU to undergo weaning from ventilatory support, rehabilitation, and other medical care needs [5].

Sleep disturbances can persist after hospital discharge and may negatively impact quality of life (QOL). In a prospective cohort study surveying survivors of acute lung injury six months after discharge, the association between insomnia symptoms and QOL (Medical Outcomes Study 36tem Short-form Health Survey, or SF-36) scores were analyzed, adjusting for post-traumatic stress disorder (PTSD) and depression [49]. Half of the subjects reported insomnia symptoms and scored worse in both mental and physical QOL. After adjusting for PTSD and depression, there remained a significant association between insomnia and physical functional impairment. Contrary to these findings, another prospective cohort study utilizing the Basic Nordic Sleep Questionnaire and the SF-36 found that the prevalence of self-reported quality of sleep did not change from the preICU period to the post-ICU period, at 6 and 12 months postdischarge [50]. The presence of concurrent diseases in the post-ICU period, however, was strongly associated with 
difficulties falling asleep, poor quality of sleep and sleep deficit, emphasizing the need for increased awareness of comorbidities as a major factor in sleep recovery after an ICU stay. Associations between sleep disturbances and Acute Physiology and Chronic Health Evaluation score, the length of stay or the treatment diagnosis were not significant.

\section{CONCLUSION}

Critical illness, various treatment modalities and the ICU environment itself can markedly impair sleep quality. Poor sleep and chronic sleep deprivation may, in turn, impair recovery with adverse effects on cognition, mental status and immune function. After discharge from the ICU, many patients will continue to experience poor sleep with potential adverse effects on functional recovery. In the busy environment of critical care, sleep quality is often overlooked. Ongoing research is beginning to demonstrate that it may no longer be prudent to disregard this essential physiological function.

\section{CONFLICT OF INTEREST}

The authors confirm that this article content has no conflict of interest.

\section{ACKNOWLEDGEMENTS}

Declared none.

\section{REFERENCES}

[1] Bihari S, McEvoy RD, Mathson E, Kim S, Woodman RJ, Bersten AD. Factors affecting sleep quality of patients in intensive care unit. J Clin Sleep Med 2012; 8: 301-7.

[2] Weinhouse GL, Schwab RJ. Sleep in the critically ill patient. Sleep 2006; 29: 707-16.

[3] Gabor JY, Cooper AB, Crombach SA, et al. Contribution of the intensive care unit environment to sleep disruption in mechanically ventilated patients and healthy subjects. Am J Respir Crit Care Med 2003; 167: 708-15.

[4] Freedman NS, Gazendam J, Levan L, Pack AI, Schwab RJ. Abnormal sleep/wake cycles and the effect of environemental noise on sleep disruption in the intensive care unit. Am J Respir Crit Care Med 2001; 163: 451-7.

[5] Fanfulla F, Ceriana P, D'Artavilla N, Trentin R, Frigerio F, Nava S. Sleep disturbances in patients admitted to a step-down unit after ICU discharge: the role of mechanical ventilation. Sleep 2011; 34: 355-62.

[6] Little A, Ethier C, Ayas N, Thanachayanont T, Jiang D, Mehta S. A patient survey of sleep quality in the intensive care unit. Minerva Anestesiol 2012; 78: 406-14.

[7] Padilha HG, Crispim CA, Zimberg IZ, et al. A line between sleep loss, glucose metabolism and adipokines. Braz J Med Biol Res 2011; 44: 992-9.

[8] Gehlbach BK, Chapotot F, Leproult R, et al. Temporal disorganization of circadian rhythmicity and sleep-wake regulation in mechanically ventilated patients receieving continuous intravenous sedation. Sleep 2012; 35: 1105-14.

[9] Le A, Friese RS, Hsu CH, Wynne JL, Rhee P, O'Keeffe T. Sleep disruptions and nocturnal nursing interactions in the intensive care unit. J Surg Res 2012; 177: 310-14.

[10] Rompaey BV, Elseviers MM, Drom WV, Fromont V, Jorens PG. The effect of earplugs during the night on the onset of delirium and sleep perception: a randomized controlled trial in intensive care patients. Crit Care 2012; 16: R73.

[11] Trompeo AC, Vidi Y, Locane MD, et al. Sleep disturbances in the critically ill patients: role of delirium and sedative agents. Minerva Anestesiol 2011;77: 604-12.

[12] Ely EW, Shintani A, Truman B, et al. Delirium as a predictor of mortality in mechanically ventilated patients in the intensive care unit. JAMA 2004; 291: 1753-62.

[13] Yu SB. Dexmedetomidine sedation in ICU. Korean J Anesthesiol 2012;6 5: 405-11.
[14] Sanders RD, Maze M. Contribution of sedative-hypnotic agents to delirium via modulation of the sleep pathway. Can J Anesth 2011; 58: $149-56$.

[15] Van Rompaey B, Elseviers MM, Van Drom W, Fromont V, Jorens PG. The effect of earplugs during the night on the onset of delirium and sleep perception: A randomized controlled trial in intensive care patients. Crit Care 2012;16(3)R73.

[16] Olofsson K, Alling C, Lundberg D, Malmros C. Abolished circadian rhythm of melatonin secretion in sedated and artificially ventilated intensive care patients. Acta Anaesthesiol Scand 2004; 48: 679-84.

[17] Mudigler G, Delle-Karth G, Koreny M, et al. Impaired circadian rhythm of melatonin secretion in sedated critically ill patients with severe sepsis. Crit Care Med 2002; 30: 536-40.

[18] Parthasarathy S, Friese R. Sleep, circadian rhythms, and critical illness. Sleep 2012; 35: 1029-30.

[19] Kryger MH, Roth T, Dement WC. Principles and practice of sleep medicine: Missouri: expert Consult 2011.

[20] Paul T, Lemmer B. Disturbance of circadian rhythms in analgosedated intensive care unit patients with and without craniocerebral injury. Chronobiol Int 2007; 24(1): 45-61.

[21] Hu R, Jiang X, Zeng Y, Chen X, Zhang Y. Effects of earplugs and eye masks on nocturnal sleep, melatonin and cortisol in a simulated intensive care unit environment. Crit Care 2010;14(2): R66.

[22] Marik PE. Surviving sepsis: going beyond the guidelines. Ann Intensive Care 2011; 1: 17. Published online 2011 June 7.

[23] Gamaldo CE, Shaikh AK, McArthur JC. The sleep-immunity relationship. Neurol Clin 2012; 30: 1313-43.

[24] Krueger JM, Clinton JM, Winters BD, et al. Involvement of cytokines in slow wave sleep. Prog Brain Res 2011; 193: 39-47.

[25] Lewy AJ, Sack RL. The dim light melatonin onset as a marker for circadian phase position. Chronobiol Inremarronnl 1989; 6: 93-102.

[26] Arjona A, Silver AC, Walker WE, Fikrig E. Immunity's fourth dimension: approaching the circadian-immune connection. Trends Immunol 2012 Sep 20. pii: S1471-4906(12)00145-7.

[27] Mazzoccoli G, Sothern RB, Greco A, et al. Time-related dynamics of variation in core clock gene expression levels in tissues relevant to the immune system. Int J Immunopathol Pharmacol 2011; 24: 869-79.

[28] Bollinger T, Leutz A, Leliavski A, et al. Circadian clocks in mouse and human CD4 T cells. PLoS ONE 2011; 6: e29801.

[29] Ackermann K, Revell VL, Lao O, Rombouts EJ, Skene DJ, Kayser M. Diurnal rhythms in blood cell populations and the effect of acute sleep deprivation in healthy young men. Sleep 2012; 35: 933$40 \mathrm{~F}$.

[30] Ruiz FS, Andersen ML, Martins RC, Zager A, Lopes JD, Tufik S. Immune alterations after selective rapid eye movement or total sleep deprivation in healthy male volunteers. Innate Immun 2010; 18: 44-54.

[31] Irwin M, Mascovich A, Gillin C, Willoughby R, Pike J, Smith TL. Partial Sleep deprivation reduced natural killer cell activity in humans. Psychosom Med 1994; 56: 493-8.

[32] Meier-Ewert HK, Ridker PM, Rifai N, et al. Effect of sleep loss on C-reactive protein, an inflammatory marker of cardiovascular risk. J Am Coll Cardiol 2004; 43: 678-83.

[33] Spiegel K, Sheridan JF, Van Cauter E. Effect of sleep deprivation on response to immunization. JAMA 2002; 288: 1471-2.

[34] Egydio F, Pires GN, Tufik S, Andersen ML. Wound healing and benzodiazepines: does sleep play a role in this relationship? Clinics 2012; 67: 827-30.

[35] Landis CA, Whitney JD. Effects of 72 hours sleep deprivation on wound healing in the rat. Res Nurs Health 1997; 20: 259-67.

[36] Besedovsky L, Lange T, Born J. Sleep and immune function. Pflugers Arch 2012; 463: 121-37.

[37] Treggiari-Venzi M, Borgeat A, Fuchs-Buder T, Gachoud JP, Suter PM. Overnight sedation with medazloam or propofol in the ICU: Effects on sleep quality, anxiety and depression. Intens Care Med 1996; 55: 1186-90.

[38] Kondili E, Alexopoulou C, Xirouchaki N, Georgopoulos D. Effects of propofol on sleep quality in mechanically ventilated critically ill patients: a physiological study. Intens Care Med 2012; 38: 164046.

[39] Huupponen E, Maksimow A, Lapinlampi P, et al. Electroencephalogram spindle activity during dexmedetomidine sedation and physiological sleep. Acta Anaesthesiol Scand 2008; 52: 289-94. 
[40] Talwar A, Liman B, Greenberg H, Feinsilver S, Vijayan VK. Sleep in the intensive care unit. Indian J Chest Dis Allied Sci 2008; 50: $151-62$.

[41] Goldman MD, Reeder MK, Muir AD, et al. Repetitive nocturnal arterial oxygen desaturation and silent myocardial ischemia in patients presenting for vascular surgery. J Am Geriatr Soc 1993; 41: 703-9.

[42] Parthasarathy S, Tobin MJ. Effect of ventilator mode on sleep quality in critically ill patients. Am J Respir Crit Care Med 2002; 166: $1423-9$

[43] Younes M, Ostrowski M, Thompson W, Leslie C, Shewchuk W. Chemical control stability in patients with obstructive sleep apnea. Am J Respir Crit Care Med 2002; 163: 1181-90.

[44] Dempsey JA, Smith CA, Przybylowski T, et al. The ventilatory responsiveness to $\mathrm{CO} 2$ below eupnoea as a determinant of ventilatory stability in sleep. J Physiol 2004; 560(1): 1-11.

[45] Lorenzi-Filho G, Genta PR, Figueiredo AC, Inoue D. CheyneStokes respiration in patients with congestive heart failure: causes and consequences. Clinics 2005; 60: 333-44.
[46] Lovering AT, Fraigne JJ, Dunin-Barkowski WL, Vidruk EH, Orem JM. Hypocapnia decreases the amount of rapid eye movement sleep in cats. Sleep 2003; 26: 961-7.

[47] Bosma K, Ferreyra G, Ambrogio C, et al. Patient-ventilator interaction and sleep in mechanically ventilated patients: pressure support versus proportional assist ventilation. Crit Care Med 2007; 35: $1048-54$

[48] Cabello B, Thille AW, Drouot X, et al. Sleep quality in mechanically ventilated patients: comparison of three ventilator modes. Crit Care Med 2008; 36: 1749-55.

[49] Parsons EC, Kross Ek, Caldwell ES et al. Post-discharge insomnia symptoms are associated with quality of life impairment among survivors of acute lung injury. Sleep Med 2012; 13: 1106-9.

[50] Orwelius L, Nordlund A, Nordlund P, Edéll-Gustafsson U, Sjöber F. Prevalence of sleep disturbances and long-term reduced healthrelated quality of life after critical care: a prospective multicenter cohort study. Crit Care 2008; 12(4): R97. doi: 10.1186/cc6973.

(C) Wang and Greenberg; Licensee Bentham Open.

This is an open access article licensed under the terms of the Creative Commons Attribution Non-Commercial License (http://creativecommons.org/licenses/by-nc/ 3.0/) which permits unrestricted, non-commercial use, distribution and reproduction in any medium, provided the work is properly cited. 